\title{
Smart Fault Diagnostics using Convolutional Neural Network and Adam Stochastic Optimization
}

\author{
Dr. Subarna Shakya, \\ Professor, \\ Department of Electronics and Computer Engineering, \\ Central Campus, Institute of Engineering, Pulchowk, \\ Tribhuvan University, \\ Pulchowk, Lalitpur, Nepal - 44600. \\ Email: drss@ioe.edu.np
}

\begin{abstract}
Navigation, aviation and several other fields of engineering extensively make use of rotating machinery. The stability and safety of the equipment as well as the personnel are affected by this machinery. Use of deep learning as the basis of intelligent fault diagnosis schemes has and investigation of other relevant fault diagnosis schemes has a large scope for development. Thorough exploration needs to be performed in deep neural network (DNN) based schemes as shallow layer network structure based fault diagnosis schemes that are currently available has several considerable limitations. The nonlinear problems may be processed during intelligent fault diagnosis using deep convolutional neural network, which is a special structure DNN. The convolutional neural network $(\mathrm{CNN})$ based scheme is emphasized in this paper. The principle and basic structure of the model are introduced. In rotating machinery, the fault diagnosis schemes using CNN are analyzed and summarized. Various CNN schemes, the potential mechanisms and performance diagnosis are analyzed. A novel smart fault diagnosis strategy is proposed while highlighting the potential aspects of existing schemes and reviewing the challenges.
\end{abstract}

Keywords: rotating machinery, smart fault diagnosis, convolution neural network, deep learning

\section{Introduction}

In the field of control engineering, fault diagnosis plays a significant role due to its comprehensive and interdisciplinary nature [1]. The reliability and safety of a mechanical 
equipment may be guaranteed by necessary preconditioning with fault diagnosis. The railway, ship building, nuclear power and aerospace domains are largely dependent on rotating machinery [2]. Frequent faults in the equipment due to the influence of non-linear factors and complexity of the system may lead to catastrophic safety accidents, loss in economy, influence the production or damage the equipment itself. The complication of failure and uncertainty during condition monitoring leads to great challenge in conducting fault diagnosis [3]. This makes fault diagnosis strategies vital for ensuring optimal operation of rotary machinery.

The traditional fault diagnosis schemes and their practical applications are studied widely by several researchers [4]. The rotating machinery and their non stationary signals are investigated using synchro extracting strategy integrated with polynomial chirplet transform technique. Local non-negative matrix factorization, in combination with optimized intrinsic characteristic scale decomposition is used for analyzing bearing fault while addressing the underdetermined blind source separation issue [5]. The hydraulic pump based faults are investigated and the fault characteristics of hydraulic systems are analyzed using improved empirical wavelet transform based feature extraction [6]. Frequency and amplitude demodulation evaluation is integrated with local mean decomposition for diagnosis of faults in planetary gearboxes. Gear tooth fault detection is performed using mapping and windowing techniques. There are several practical limitations in these traditional diagnostic schemes due to their high level of dependency on people and their experience [7].

\section{Related Works}

The engineering field has an irresistible trend towards smart systems. This has led to the development of smart fault diagnosis systems as well [8]. Traditional machine learning schemes are used for developing smart fault diagnosis based models by several researchers. Compound fault diagnosis is performed by combining the Empirical Mode Decomposition (EMD) based on non-Naïve Bayesian classier for developing a smart system. With changing severity levels and fault positions, the rolling element bearing and its fault features were analyzed by integrating nearest neighbor classier and symbolic aggregate approximation [9]. The robustness and reliability presented by this technique is found to be favorable in various applications. The faults in roller bearings are identified by achieving feature extraction through the integration of wavelet package transform and statistic filter along with moving peak-hold 
strategy [7]. The wind turbines are investigated for gearbox faults with the help of Shannon wavelet support vector machine (SVM). SVM, ANN and random forest based smart fault diagnosis approaches are compared and analyzed for rotating machinery. Fault diagnosis is performed by employing sparse representation based on SVM [10]. The rotary machinery fault may also be diagnosed by combining SVM along with optimized quantum genetic algorithm. Improved SVM technique is examined to address multiple failure issues. Loss of essential data and external interference may influence the diagnostic characteristics by the smart fault diagnosis schemes in comparison to the traditional techniques despite their ability to overcome certain complex nonlinear problems [11].

Complex classification issues may be addressed in a superior manner with more abstract representations and effective feature distinguishing due to the automatic learning ability of deep neural network (DNN) that helps it to overcome the drawbacks of shallow neural network [12]. This has caused several researchers to be intrigued in the field of machinery and DNN based fault diagnostic techniques. In order to monitor the mechanical conditions, deep belief network (DBN) is employed. Feature learning may be performed for diagnosis of bearing faults by combining Gaussian visible units with convolutional DBN [13]. The drawbacks in the conventional stacked autoencoder (SAE) may be overcome for gearbox and bearing fault diagnosis with enhanced DNN with batch normalization. Fault diagnosis is performed on rolling bearing using transfer learning and sparse stacked denoising autoencoder (SDAE) based technique to overcome the shortage of running data under various settings. The effectiveness of training is further enhanced by integrating pruning method with the SDAE model. Fine tuning operation is performed on diagnosis of gearbox faults with negative correlation learning and selective SDAE [14]. Distinguishing fault features are obtained by combining weighted wavelet coefficients with deep residual network for planetary gearbox fault diagnosis. Spatial subsampling, weight sharing and local connection are some of the special characteristics of convolution neural network $(\mathrm{CNN})$ that contributes to the benefits in smart fault diagnosis when compared to the DNN based techniques [15].

\section{Proposed Work}

A CNN based smart fault diagnostic technique with progressive strategy is presented in this paper for fault diagnosis in machinery. In machinery applications, high diagnostic 
performance and positive classification effect are achieved using CNN based diagnostic techniques as exhibited in several researches. Pump, gearbox, gear and other bearings are used extensively in rotary machinery. This analysis is beneficial to their function on a large scale. The vibration data acquired form these machinery varies due to the ambient noise caused by the variation in working loads despite the numerous types of rotating machinery. The results of prediction during final detection are affected at various degrees. Practical and academic researches on fault diagnosis have pointed out this challenge.

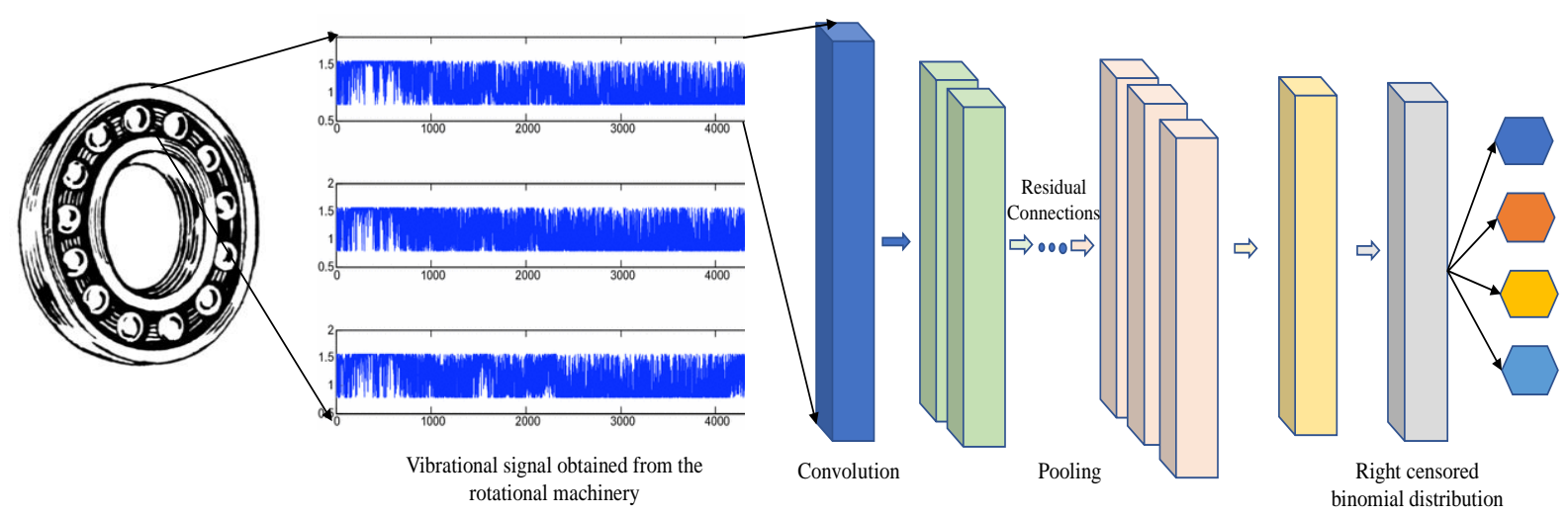

Fig. 1. Proposed modified CNN framework for smart fault diagnosis

Figure 1 provides the proposed modified CNN framework for smart fault diagnosis in rotary parts of machinery. Automatic feature learning is performed using $\mathrm{CNN}$ due to its special characteristics and structure. Complex preprocessing stages and expert experience dependence negatively impacts the smart diagnostic schemes based on CNN. The performance of classification and promotion of various techniques are addressed by varying the CNN design. One and two dimensional (1D and 2D) CNN are used commonly in smart fault diagnosis of machinery using spectral images based on the raw vibration signals. Based on CNN, discussion and analysis of each rotating machinery is performed. Based on the classification discrimination and diagnostic accuracy, smart fault diagnosis techniques are evaluated for the potential mechanisms in enhancement of diagnosis. 


\section{Results and Discussion}

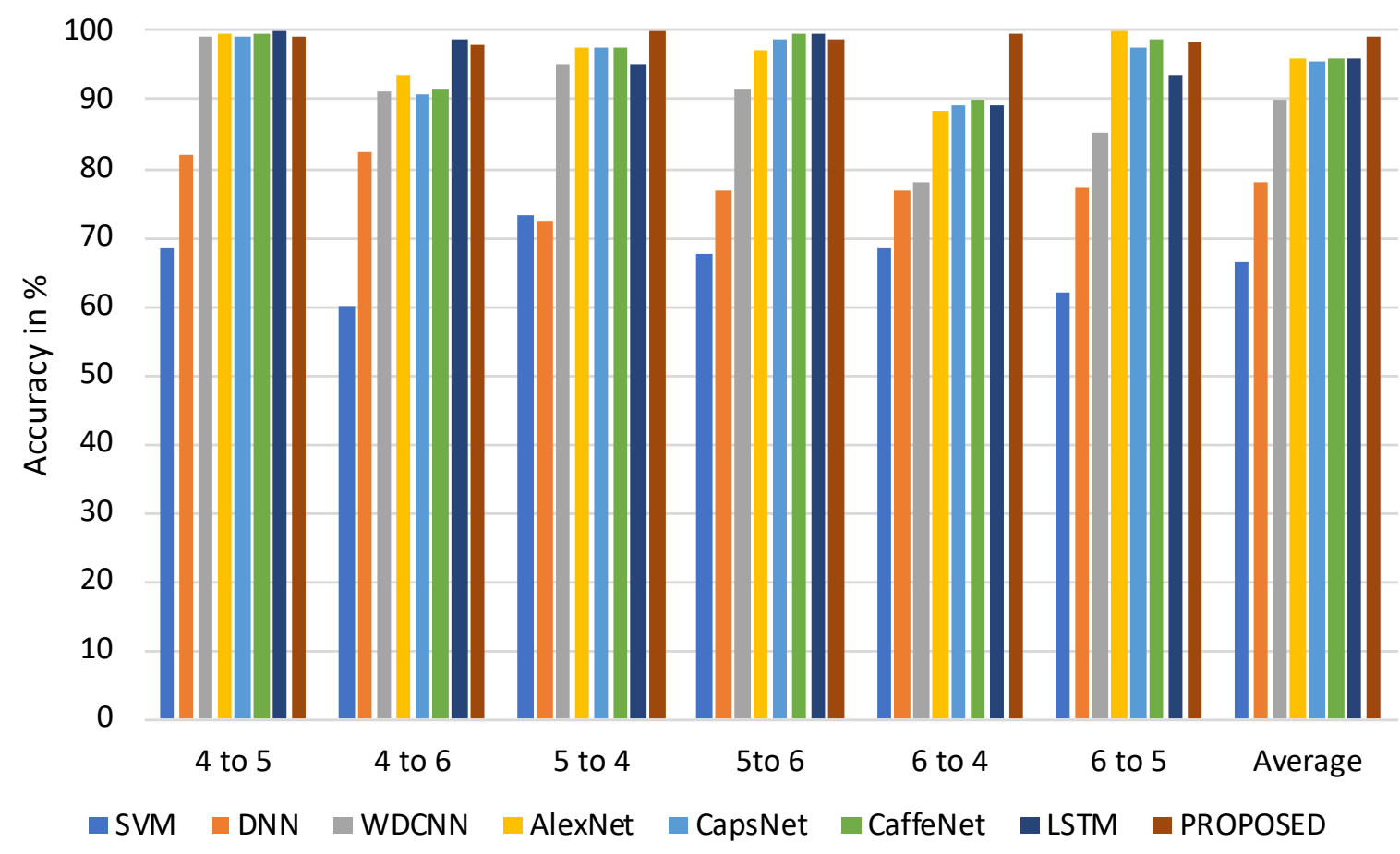

Fig. 2. Comparison of the proposed scheme with existing models

When compared to the existing schemes like SVM, DNN, WDCNN, AlexNet, CapsNet, CaffeNet and LSTM, the proposed CNN based smart fault diagnosis technique offers improved accuracy for fault diagnosis in rotary machinery. Figure 2 represents the comparison of accuracy of the aforementioned techniques. In machinery based applications, improved diagnostic performance and classification effect is observed using CNN based diagnostic schemes. Pump, gearbox, gear and bearing in rotary machinery are analyzed in an extensive manner by applying these diagnostic schemes to evaluate their performance. The acquired vibration data is affected due to the ambient noise and interference caused by the changing working loads irrespective of the type of rotating machinery. The prediction and detection outputs are influenced at multiple degrees. In practical and academic research related to fault diagnosis, this challenge is evident.

Automatic feature learning is a powerful ability presented by CNN due to its exclusive characteristics and structure. The complex preprocessing steps and expert experience dependency are reduced largely by smart diagnostic schemes using CNN. The classification performance of these schemes and their promotion is performed by addressing multiple 
Journal of Soft Computing Paradigm (JSCP) (2021)

Vol.03/ No.01

Pages: $38-46$

http://irojournals.com/jscp/

DOI: $\underline{\text { https://doi.org/10.36548/jscp.2021.1.005 }}$

problems using altered CNN designs. 1D and 2D CNN structures are used in smart fault diagnosis in rotary machines using spectral images and raw vibration signals. The 1D and 2D $\mathrm{CNN}$ is used as the basis for analysis of various rotating machinery. Further, classification discrimination and diagnostic accuracy perspectives are used for analysis of smart fault diagnostic schemes and the potential mechanisms are enhanced diagnostically if required.

Complete data preprocessing is performed using cyclic spectral coherence considering the bearing faults developed caused by potential repeated effects. The recognition capability is enhanced in a distinct manner based on LeNet-5 integrated in the CNN based diagnostic model. The ability of generalization is promoted to overcome the effects of imbalanced data distribution using group normalization technique. The diagnostic accuracy of $99 \%$ is achieved using this approach which is remarkable when compared to the other technique. Under certain varying working conditions, over $99 \%$ accuracy has also been achieved. Figure 3 provides the visualization of various features obtained at the convolution layer of the proposed model.

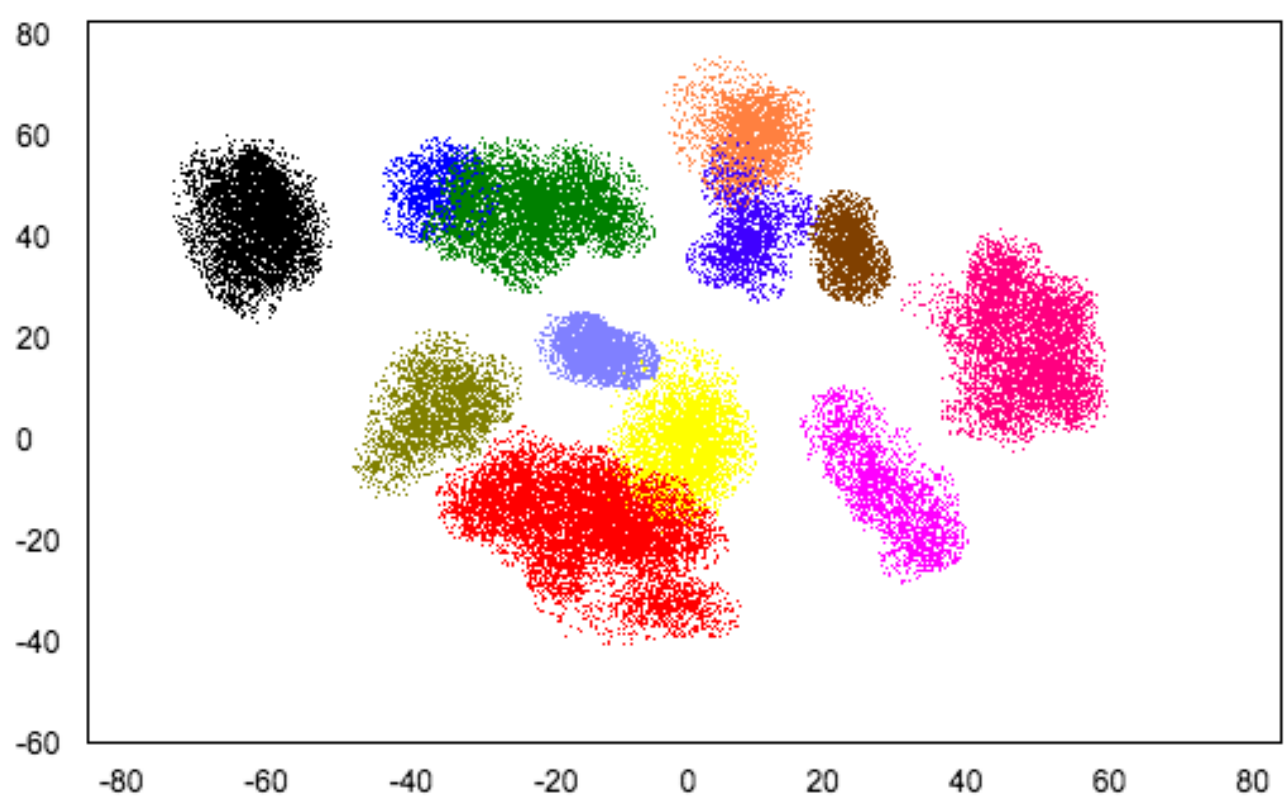

Fig. 3. Visualization of various features at convolution layer

The bearing fault diagnosis scheme using CNN is an end-to-end model where performance reduction caused by the environmental noise based interference and changing work load is addressed in a significant manner. This work highlights the special training 
technique used for the performance improvement. Complex preprocessing is overcome by employing the framework with small batch training and dropout schemes. The loss function is minimized eliminating the regularization term by training the model using Adam Stochastic optimization algorithm. Over $99.5 \%$ classification accuracy is obtained with an SNR of 8 . The features extracted from each CNN layer are evaluated to investigate the potential mechanism while visualizing and understanding the effect of classification. Minimum entropy deconvolution is used for constructing a novel CNN technique for exploring axial piston pump based unknown failure mechanism. The classification performance and feature learning stability is enhanced by preprocessing the raw signals in this technique. This model also demonstrates improved classification accuracy when compared to other techniques. Figure 4 represents the simulation verification and $3 \mathrm{D}$ representation of feature learning process. These results show that the proposed technique is advantageous over other diagnostic schemes discussed in this paper.

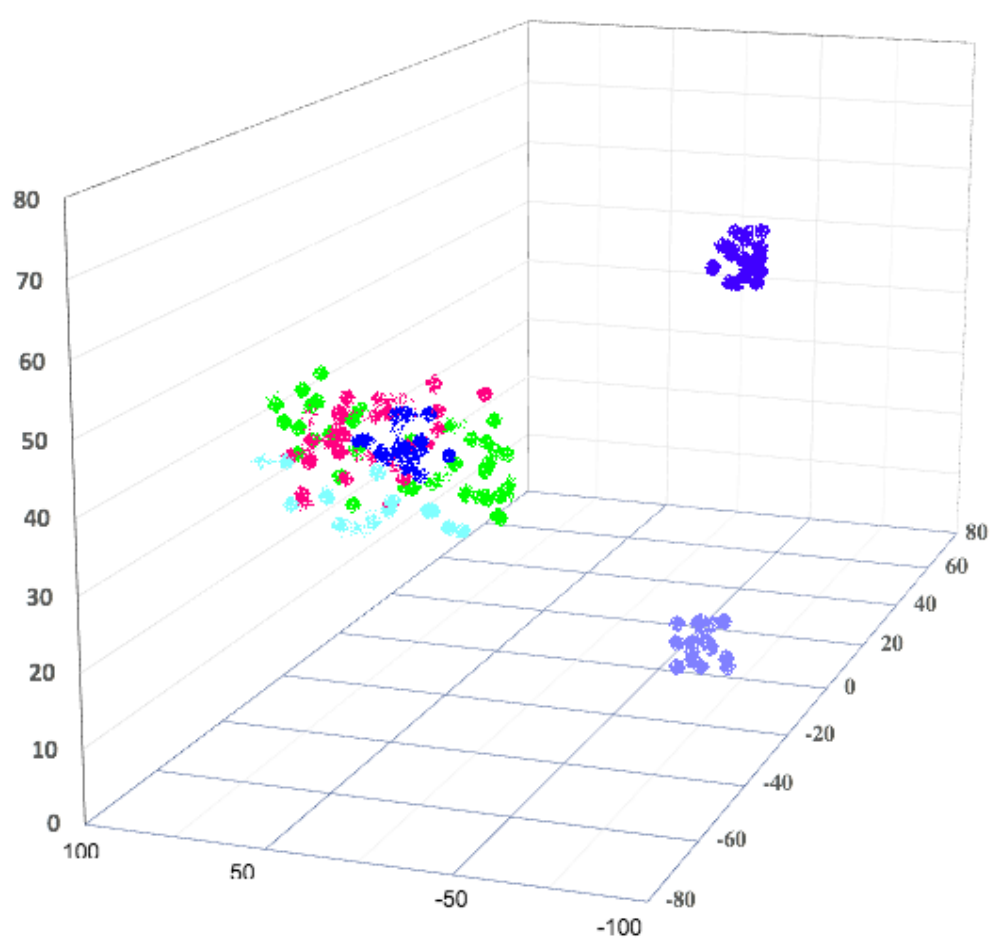

Fig. 4. Simulation verification and 3D representation of feature learning process

\section{Conclusion}

Modern DNN based techniques and traditional shallow network schemes are used for smart fault diagnosis. Mechanical big data may be handled in an efficient manner with the help of the powerful deep learning technology due to its potency in processing big data. The feature 
Journal of Soft Computing Paradigm (JSCP) (2021)

Vol.03/ No.01

Pages: $38-46$

http://irojournals.com/jscp/

DOI: $\underline{\text { https://doi.org/10.36548/jscp.2021.1.005 }}$

extraction and potential automatic learning features of DNN based technologies perform excellent fault classification. Pump, gear and gearbox, bearing as well as rotary machinery and the use of $\mathrm{CNN}$-based schemes in them are surveyed primarily. The recent relative researches and their classification effects are analyzed and contrasted with other existing schemes. Several desirable achievements have been made by several researchers in this domain. However, thorough research and thinking are required to overcome more challenging issues.

Incomplete feature extraction may be caused by improper classification performance. Extracted feature representation may be limited due to the vulnerability of essential information. As single fault processing is addressed by several schemes, it is essential to explore the ability to generalize these techniques. Another prominent drawback of CNN-based schemes is that, it is unclear regarding the suitable scheme for overcoming complexed compound fault. Several approaches are fused together and new techniques are developed for overcoming these drawbacks. Generalization of these mechanisms are challenging and enhancement of intrinsic mechanisms are unclear. Comparison of the fusion technique with the traditional schemes is performed. Future work is focused on building a prospective strategy to overcome more composite faults in machinery using $\mathrm{CNN}$ based fault diagnosis schemes.

\section{References}

[1] Ou, M., Wei, H., Zhang, Y., \& Tan, J. (2019). A dynamic adam based deep neural network for fault diagnosis of oil-immersed power transformers. Energies, 12(6), 995.

[2] Hoang, D. T., \& Kang, H. J. (2019). Rolling element bearing fault diagnosis using convolutional neural network and vibration image. Cognitive Systems Research, 53, 42 50.

[3] Lu, X., Lin, P., Cheng, S., Lin, Y., Chen, Z., Wu, L., \& Zheng, Q. (2019). Fault diagnosis for photovoltaic array based on convolutional neural network and electrical time series graph. Energy Conversion and Management, 196, 950-965.

[4] Janeera, D. A., Amudhavalli, P., Sherubha, P., Sasirekha, S. P., Raj, P. A. C., \& Sitharthan, R. (2020, September). Visualization and Classification of Diseases using Deep Learning Based Convolution Neural Network. In IOP Conference Series: Materials Science and Engineering (Vol. 937, No. 1, p. 012053). IOP Publishing. 
Journal of Soft Computing Paradigm (JSCP) (2021)

Vol.03/ No.01

Pages: $38-46$

http://irojournals.com/jscp/

DOI: $\underline{\text { https://doi.org/10.36548/jscp.2021.1.005 }}$

[5] Sun, W., Yao, B., Zeng, N., Chen, B., He, Y., Cao, X., \& He, W. (2017). An intelligent gear fault diagnosis methodology using a complex wavelet enhanced convolutional neural network. Materials, 10(7), 790.

[6] Zhang, W., Peng, G., \& Li, C. (2017). Rolling element bearings fault intelligent diagnosis based on convolutional neural networks using raw sensing signal. In Advances in Intelligent Information Hiding and Multimedia Signal Processing (pp. 77-84). Springer, Cham.

[7] Zhang, W., Peng, G., \& Li, C. (2017). Bearings fault diagnosis based on convolutional neural networks with 2-D representation of vibration signals as input. In MATEC web of conferences (Vol. 95, p. 13001). EDP Sciences.

[8] Huang, R., Liao, Y., Zhang, S., \& Li, W. (2018). Deep decoupling convolutional neural network for intelligent compound fault diagnosis. IEEE Access, 7, 1848-1858.

[9] Chen, Z., Gryllias, K., \& Li, W. (2019). Intelligent fault diagnosis for rotary machinery using transferable convolutional neural network. IEEE Transactions on Industrial Informatics, 16(1), 339-349.

[10] Jiao, J., Zhao, M., Lin, J., \& Liang, K. (2020). A comprehensive review on convolutional neural network in machine fault diagnosis. Neurocomputing, 417, 36-63.

[11] Xu, X., Tao, Z., Ming, W., An, Q., \& Chen, M. (2020). Intelligent monitoring and diagnostics using a novel integrated model based on deep learning and multi-sensor feature fusion. Measurement, 165, 108086.

[12] Ma, P., Zhang, H., Fan, W., Wang, C., Wen, G., \& Zhang, X. (2019). A novel bearing fault diagnosis method based on 2D image representation and transfer learning-convolutional neural network. Measurement Science and Technology, 30(5), 055402.

[13] Sungheetha, A., \& Sharma, R. (2020). Transcapsule model for sentiment classification. Journal of Artificial Intelligence, 2(03), 163-169.

[14] Bashar, A. (2019). Survey on evolving deep learning neural network architectures. Journal of Artificial Intelligence, 1(02), 73-82.

[15] Smys, S., Chen, J. I. Z., \& Shakya, S. (2020). Survey on Neural Network Architectures with Deep Learning. Journal of Soft Computing Paradigm (JSCP), 2(03), 186-194. 